\title{
Blessures liées à une chute chez les aînés canadiens entre 2005 et 2013 : une analyse de l'Enquête sur la santé dans les collectivités canadiennes
}

\author{
M. T. Do, Ph. D. (1); V. C. Chang, M.H.P. (2); N. Kuran, M.A. (3); W. Thompson, M. Sc. (1)
}

Cet article a fait l'objet d'une évaluation par les pairs.

\section{Résumé}

Introduction : Nous décrivons l'épidémiologie et les tendances des blessures liées à une chute chez les aînés canadiens de 65 ans et plus selon le sexe et l'âge, ainsi que les circonstances et les conséquences de ces blessures.

Méthodologie : Nous avons utilisé des données représentatives tirées des échantillons de 2005, de 2009-2010 et de 2013 de l'Enquête sur la santé dans les collectivités canadiennes afin de calculer le nombre et les taux de blessures liées à une chute pour chaque année d'enquête. Nous avons combiné, dans la mesure du possible, les données d'au moins deux échantillons afin d'estimer la proportion de blessures liées à une chute par type de blessure, partie du corps affectée, type d'activité et type de traitement.

Résultats : Le taux de blessures liées à une chute chez les aînés est passé de 49,4 à 58,8 pour 1000 personnes entre 2005 et 2013, période durant laquelle le nombre de blessures liées à une chute a de façon générale augmenté de $54 \%$. Les femmes ont présenté des taux plus élevés que les hommes pour toutes les années d'enquête, ces taux augmentant avec l'âge. La tendance à la hausse dans les taux de blessures liées à une chute était plus marquée chez les femmes et les plus jeunes. Les cassures et les fractures (37\%) ont été les blessures les plus courantes, et l'épaule et le bras (16\%) ont été les parties du corps les plus couramment affectées. Plusieurs blessures liées à une chute se sont produites alors que la personne marchait sur une surface non enneigée ou glacée (45\%). Plus de 70 \% des aînés qui ont cherché à obtenir des soins médicaux pour leur blessure se sont rendus au service d'urgence d'un hôpital.

Conclusion : Compte tenu de l'augmentation du nombre et des taux de blessures liées à une chute au fil du temps, la surveillance des tendances et des profils de ces dernières demeure nécessaire.

Mots-clés : chutes, blessures accidentelles, aînés, Canada

\section{Introduction}

Chaque année, environ un aîné sur trois fait une chute ${ }^{1-5}$. En 2008-2009, $20 \%$ des Canadiens âgés de 65 ans ou plus vivant dans la collectivité ont déclaré avoir fait une chute l'année précédente ${ }^{6}$. Cette prévalence augmente avec l'âge, passant de
$17 \%$ chez les 65 à 69 ans à $27 \%$ chez les 85 ans et plus ${ }^{6}$.

Les chutes sont également la principale cause d'hospitalisation attribuable à une blessure chez les aînés canadiens ${ }^{7}$. La durée moyenne du séjour à l'hôpital qui leur est associée est d'environ 3 semaines,

\section{Principales constatations}

- Entre 2005 et 2013, le taux de blessures liées à une chute chez les aînés canadiens âgés de 65 ans ou plus est passé de 49,4 à 58,8 pour 1000 personnes.

- Les blessures liées à une chute sont plus fréquentes chez les femmes et les groupes plus âgés.

- Entre 2005 et 2013, une augmentation significative du taux de blessures liées à une chute a été observée chez les femmes et les moins de 75 ans.

- Les fractures ont représenté $37 \%$ de l'ensemble des blessures liées à une chute, ce qui souligne l'importance de la santé des os.

- Nous avons besoin de plus d'années de données d'enquête pour évaluer les tendances à long terme en ce qui concerne les blessures liées à une chute chez les aînés.

ce qui excède de $75 \%$ la durée moyenne de séjour pour toutes les causes d'hospitalisation combinées ${ }^{7}$. Les blessures liées à une chute sont associées à une forte incapacité, à une réduction de la mobilité et de l'autonomie, à une plus grande probabilité d'être admis dans une maison de soins infirmiers et à un risque accru de décès prématuré $^{8,9}$. Les chutes sont responsables de $95 \%$ des fractures de la hanche chez les adultes plus âgés ${ }^{10}$, et elles entraînent le décès dans plus de $20 \%$ des $\operatorname{cas}^{11}$.

Même si elles ne provoquent pas de blessure, les chutes peuvent avoir des

Rattachement des auteurs :

1. Division de la surveillance et de l'épidémiologie, Agence de la santé publique du Canada, Ottawa (Ontario), Canada

2. École de santé publique Dalla Lana, Université de Toronto, Toronto (Ontario), Canada

3. Unité des politiques sur les aînés, Division des enfants, des aînés et du développement sain, Agence de la santé publique du Canada, Ottawa (Ontario), Canada

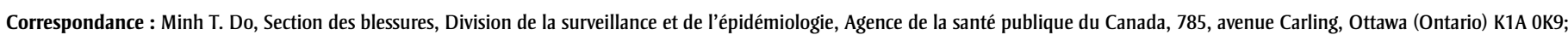
tél.: 613-946-6958; téléc. : 613-941-2057; courriel : minh.t.do@phac-aspc.gc.ca 
conséquences psychologiques à long terme, comme la dépression, la peur de chuter et la perte de confiance, qui entraînent à leur tour des restrictions dans les activités quotidiennes et sociales, puis une baisse de la santé et des fonctions ainsi qu'une augmentation du risque de chute ultérieure ${ }^{12-14}$.

De plus, les blessures liées à une chute constituent un fardeau économique pour le système de santét ${ }^{15-18}$ : au Canada, les coûts médicaux directs associés aux chutes chez les aînés étaient estimés à plus de 2 milliards de dollars en $2004^{19}$. On s'attend à une augmentation substantielle de ces coûts, étant donné que le nombre de Canadiens âgés de 65 ans ou plus devrait plus que doubler, passant de 5 millions (15\%) en $2011^{20}$ à près de 11 millions $(25 \%)$ en $2036^{21}$.

En avril 2014, l'Agence de la santé publique du Canada a publié son deuxième rapport sur les chutes chez les aînés ${ }^{7}$. Ce rapport a fourni un aperçu des facteurs de risque de chute et a présenté les estimations nationales du nombre de blessures liées à une chute, estimations calculées à partir de données d'enquête ainsi que de données sur les hospitalisations et la mortalité. Les données recueillies dans le cadre de l'Enquête sur la santé dans les collectivités canadiennes (ESCC) ont ainsi montré que le nombre d'aînés ayant déclaré avoir subi une blessure attribuable à une chute était passé d'environ $179 \quad 000$ à $256 \quad 000$ (augmentation de $43 \%$ ) entre 2003 et $2009-2010^{7}$. De plus, le taux de blessures liées à une chute s'est révélé significativement plus élevé chez les femmes que chez les hommes à chacune des années d'enquête ${ }^{7}$. L'accessibilité aux nouvelles données de l'ESCC de 2013 justifie une actualisation des analyses des blessures liées à une chute chez les aînés canadiens.

Cette étude vise (1) à fournir les plus récentes estimations nationales du nombre et du taux de blessures autodéclarées par suite d'une chute, par sexe et par groupe d'âge, et à en évaluer les tendances entre 2005 et 2013, (2) à comparer la distribution des principales caractéristiques entre les aînés victimes d'une blessure liée à une chute et les autres aînés et (3) à décrire les caractéristiques des blessures liées à une chute, en particulier leur nature et les circonstances dans lesquelles elles sont survenues, de même que l'utilisation des services de santé qui leur est associée.

\section{Méthodologie}

\section{Source des données}

Nous avons utilisé des données représentatives tirées des ESCC menées en 2005, en 2009-2010 (combinées) et 2013. L’ESCC est une enquête transversale qui vise à recueillir auprès de la population canadienne des renseignements sur l'état de santé, l'utilisation des services de santé et les déterminants de la santé. Utilisant une méthode stratifiée d'échantillonnage en grappes, l'ESCC vise les personnes de 12 ans et plus occupant un logement privé dans les dix provinces et les trois territoires du Canada, à l'exclusion de celles vivant dans les réserves des Premières Nations et sur les terres de la Couronne, des résidents d'établissements, des membres à plein temps des Forces canadiennes et des résidents de certaines régions éloignées. Trois bases de sondage ont été utilisées pour sélectionner l'échantillon de ménages, $99 \%$ provenant d'une base aréolaire ou d'une liste de numéros de téléphone et le $1 \%$ restant d'une base constituée au moyen d'un système d'appel aléatoire. Toutes les enquêtes ont été menées à l'aide d'une méthode d'entrevue assistée par ordinateur, en personne ou téléphonique. Les taux de réponse aux ESCC de 2005, de 2009-2010 et de 2013 ont été de respectivement $78,9 \%, 72,3 \%$ et $66,8 \%$. Depuis 2007, au lieu de recueillir les données sur une année et de publier les données aux deux ans, les responsables de l'ESCC procèdent à la collecte des données de façon continue et les publient chaque année (ou tous les deux ans sous forme d'un fichier regroupant deux années de données).

Les données des enquêtes de 2007-2008 et de 2011-2012 n’ont pas été incluses dans notre analyse étant donné que, pour ces années, les renseignements sur les blessures n'ont été recueillis que pour certaines provinces ou certains territoires (Colombie-Britannique et Nouvelle-Écosse en 2007-2008, Alberta et Territoires du Nord-Ouest en 2011-2012). De plus, comme la collecte des données de l'ESCC de 2014 était toujours en cours au moment de notre analyse, les estimations les plus récentes ont été effectuées à l'aide du fichier de données annuelles de 2013, dont l'échantillon était plus réduit que ceux de 2005 et de 2009-2010. Une description détaillée de l'ESCC, de son contexte et de sa méthodologie a été publiée ailleurs ${ }^{22-24}$.

Nos échantillons proviennent de fichiers partagés de l'ESCC contenant les dossiers de tous les répondants qui ont accepté que leurs données soient transmises à l'Agence de la santé publique du Canada, soit environ $95 \%$ de l'échantillon complet de chaque année d'enquête. De très légères différences dans la distribution des variables ont été observées entre le fichier partagé et le fichier maître contenant l'échantillon complet de l'ESCC ${ }^{24}$. Pour les besoins de cette étude, seuls les répondants âgés de 65 ans ou plus ont été inclus dans les échantillons (soit 26188 en 2005, 28379 en 2009-2010 et 17290 en 2013).

\section{Blessure liée à une chute}

Le module sur les blessures de l'ESCC incluait une question invitant les répondants à indiquer s'ils avaient subi une blessure suffisamment grave pour limiter leurs activités normales (p. ex. une fracture, une coupure profonde, une brûlure grave ou une entorse à la cheville) au cours de l'année précédente. Ceux qui répondaient « oui » à la question « $\mathrm{Au}$ cours des 12 derniers mois, vous êtes-vous blessé? " étaient invités à décrire leur blessure la plus grave ainsi qu'à préciser si cette blessure avait été causée par une chute. Ceux qui répondaient « oui » étaient considérés comme ayant subi une blessure attribuable à une chute. Les renseignements sur le type de blessure, la partie du corps affectée, le type d'activité associé à la chute et le type de traitement reçu dans les 48 heures suivant la blessure ont été recueillis à partir d'une liste prédéfinie de réponses aux questions.

\section{Caractéristiques des répondants}

Les caractéristiques des répondants étaient :

- leur sexe;

- leur groupe d'âge (65 à 69 ans, 70 à 74 ans, 75 à 79 ans, 80 à 84 ans, 85 à 89 ans et 90 ans et plus); 
- leur situation familiale (marié(e)/ conjoint(e) de fait, veuf ou veuve, séparé(e)/divorcé(e) et célibataire ou jamais marié(e));

- leur plus haut niveau de scolarité atteint (études secondaires non terminées, diplôme d'études secondaires, études postsecondaires non terminées et diplôme d'études postsecondaires);

- le revenu de leur ménage (moins de 15000 \$, de 15000 \$ à 29999 \$, de 30000 \$ à 49999 \$, de 50000 \$ à 79999 \$ et 80000 \$ et plus).

Pour faciliter la comparaison, nous avons classé ces variables conformément au rapport préparé par l'Agence de la santé publique du Canada ${ }^{7}$. Les répondants pour lesquels il manquait des données (c.-à-d. qui avaient indiqué " Ne sait pas " ou « Refus de répondre ») à chacune des variables ont été placés dans une catégorie distincte, sauf en ce qui concerne le revenu du ménage, catégorie pour laquelle les données manquantes (environ $20 \%$ ) ont été imputées par Statistique Canada selon la méthode du plus proche voisin ${ }^{25}$.

\section{Analyses statistiques}

Nous avons analysé, à l'aide de statistiques descriptives, la distribution des caractéristiques individuelles en fonction, dans chaque échantillon d'enquête, des réponses touchant les blessures liées à une chute. Le test du khi carré de Rao-Scott, qui est l'équivalent d'un test du khi carré de Pearson ajusté pour les effets du plan de sondage $^{6}$, a été utilisé pour vérifier les différences dans la distribution des caractéristiques entre les aînés victimes d'une blessure liée à une chute et les autres aînés. Nous avons également calculé la fréquence et la prévalence (nombre de personnes déclarant avoir subi une blessure attribuable à une chute au cours de l'année précédente, pour 1000 personnes) des blessures liées à une chute pour chaque échantillon d'enquête, puis effectué une stratification supplémentaire par sexe et par groupe d'âge. Pour l'examen des tendances, nous avons utilisé des tests $\mathrm{Z}$ visant à évaluer les différences de taux entre échantillons d'enquête successifs, ainsi qu'entre les échantillons de 2005 et de 2013. Tous les tests étaient bilatéraux, et nous avons considéré comme statistiquement significative une valeur $p$ inférieure à 0,05 . Compte tenu des effectifs peu élevés dans certaines catégories, les données d'au moins deux des trois échantillons d'enquête ont été combinées $^{27}$ pour calculer la proportion de blessures liées à une chute par type de blessure, par partie du corps affectée, par type d'activité et par type de traitement reçu.

Pour tenir compte du concept d'échantillonnage complexe de l'ESCC (rajustements dans les cas de non-réponse, sous-dénombrement et stratification a posteriori, etc.) et obtenir des estimations représentatives de la population canadienne, nous avons utilisé des poids d'échantillonnage dans toutes les analyses $^{24}$. Les estimations de la variance et des intervalles de confiance (IC) à $95 \%$ et des coefficients de variation (CV), ont été calculées à l'aide de la méthode bootstrap et du programme BOOTVAR 3.2 développé par Statistique Canada ${ }^{28}$. La méthode bootstrap consiste à sélectionner successivement des échantillons aléatoires $(n=500)$ de l'ensemble de données de l'ESCC, puis à recalculer les poids d'échantillonnage pour chaque sous-échantillon sélectionné et à effectuer la stratification des poids pour chaque strate afin d'obtenir les poids bootstrap finaux ${ }^{24,28}$. Les estimations sont présentées en respectant les lignes directrices relatives à la variabilité d'échantillonnage de Statistique Canada ${ }^{24}$ : celles avec un CV entre $16,6 \%$ et $33,3 \%$ sont à interpréter avec prudence en raison de leur variabilité élevée et celles avec un CV supérieur à 33,3\% n'ont pas été incluses en raison de leur variabilité extrême.

Toutes les analyses ont été effectuées à l'aide de la version $9.3 \mathrm{du}$ programme SAS (SAS Institute Inc., Cary, Caroline du Nord, É.-U.).

\section{Résultats}

Les échantillons de 2005, de 2009-2010 et de 2013 de l'ESCC étaient de respectivement 26188,28379 et 17290 répondants âgés de 65 ans ou plus. Ils sont représentatifs d'une population totale d'aînés canadiens vivant dans la collectivité d'environ 3,9 millions en 2005 et 5,1 millions en 2013.

Le tableau 1 présente la distribution des caractéristiques des répondants victimes d'une blessure liée à une chute au cours des 12 mois précédant l'enquête et celles des autres répondants dans chacun des trois échantillons de l'ESCC. Pour toutes les années d'enquête, les variations étaient notables selon le sexe, l'âge et la situation familiale $(p<0,05)$ : comparativement aux aînés ayant déclaré ne pas avoir subi de blessure liée à une chute, ceux ayant indiqué avoir subi une blessure liée à une chute étaient plus nombreux à être de sexe féminin, à être plus âgés et à être veufs, et moins nombreux à être mariés. Dans les trois échantillons, nous n'avons observé aucune différence statistiquement significative en fonction du niveau de scolarité ou du revenu du ménage.

\section{Taux et tendances}

La figure 1 présente une estimation du nombre de blessures liées à une chute chez les aînés canadiens pour toutes les années d'enquête et des taux associés à ces blessures. En 2005, 194135 Canadiens âgés de 65 ans ou plus ont déclaré avoir subi une blessure en raison d'une chute au cours des 12 mois précédant l'enquête. Ils ont été 256011 en 2009-2010 et 299769 en 2013 à le faire, ce qui représente une augmentation globale de $54 \%$ entre 2005 et 2013 . Une tendance à la hausse similaire a été observée dans le taux de blessures liées à une chute, lequel est passé de 49,4 (IC à $95 \%: 45,5$ à 53,3) pour 1000 personnes en 2005, à 57,5 (IC à $95 \%$ : 52,6 à 62,3) pour 1000 personnes en $2009-2010$ et à 58,8 (IC à $95 \%$ : 53,0 à 64,6) pour 1000 personnes en 2013. L'estimation du taux était considérablement plus élevée en 2013 qu'en 2005 $(p=0,01)$. Cependant, alors qu'une augmentation statistiquement significative du taux a été observée entre 2005 et 2009-2010 (8,1 pour 1000 personnes, $p=0,01$ ), celuici a semblé se stabiliser, avec seulement une légère augmentation, non significative, entre 2009-2010 et 2013 (1,3 pour 1000 personnes, $p=0,72$ ).

La figure 2 présente les taux de blessures liées à une chute par sexe et par année d'enquête. D'après les IC ne se chevauchant pas, pour toutes les années d'enquête, les femmes présentaient des taux significativement plus élevés de blessures liées à une chute, les ratios 
TABLEAU 1

Caractéristiques des aînés (65 ans et plus) et blessures liées à une chute, Canada, ESCC 2005, 2009-2010 et 2013

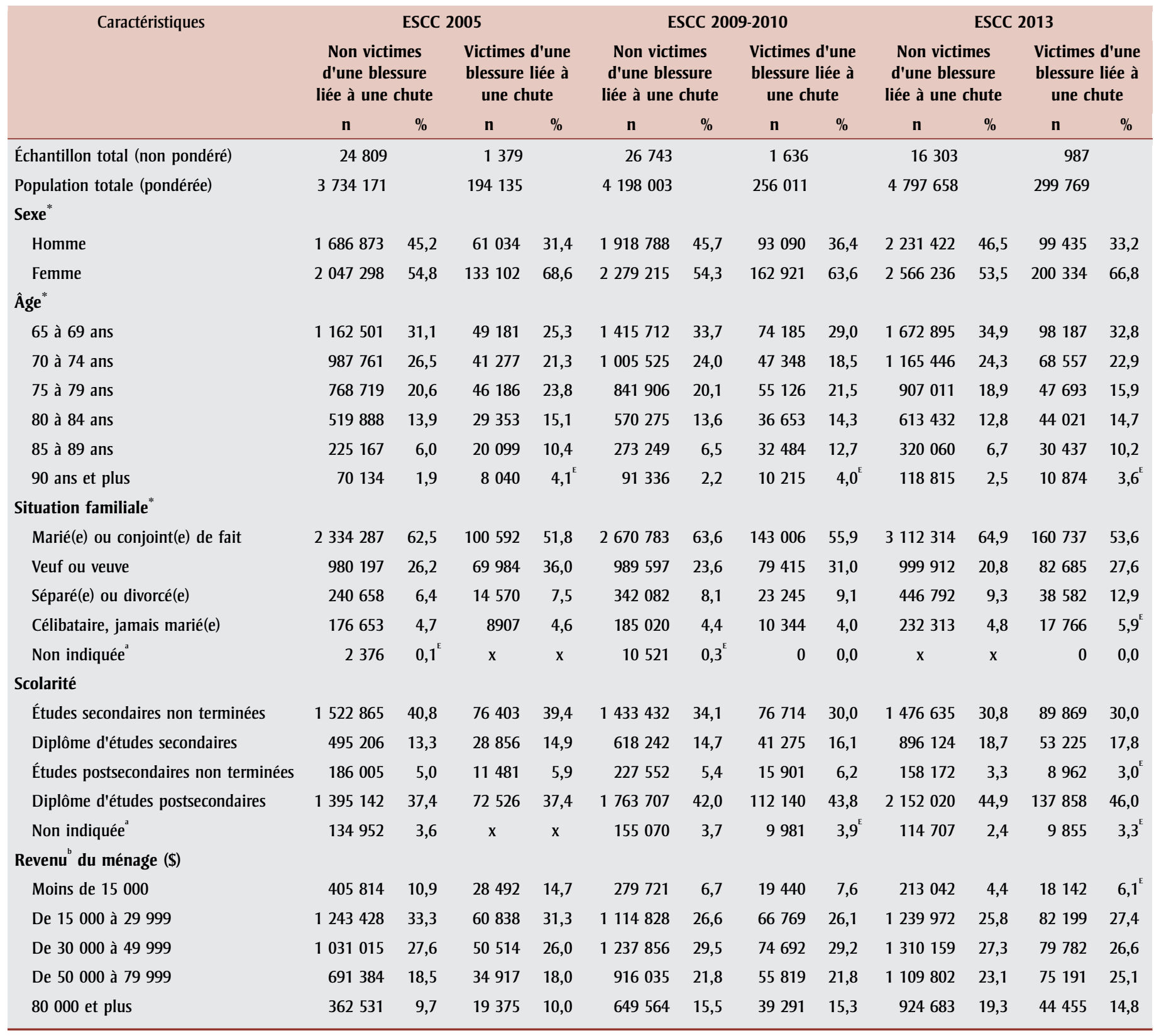

Source : Enquête sur la santé dans les collectivités canadiennes (2005, 2009-2010 et 2013), Statistique Canada.

Abréviation : ESCC, Enquête sur la santé dans les collectivités canadiennes.

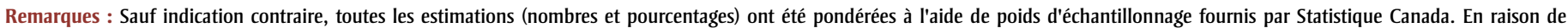
l'arrondissement, il se peut que la somme ne corresponde pas aux effectifs totaux de population et que les pourcentages ne totalisent pas $100 \%$.

a « Non indiquée » englobe toutes les réponses « Ne sait pas », « Refus de répondre » et « Non précisée ».

b Les données manquantes sur le revenu du ménage ont été imputées selon la méthode du plus proche voisin ${ }^{25}$.

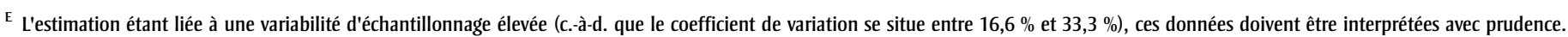
« $\mathrm{x}$ » indique que les données ne respectent pas les normes en matière de déclaration en raison de leur variabilité extrême (coefficient de variation supérieur à 33,3 \%).

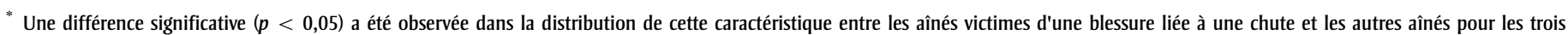
échantillons de l'étude.

femmes-hommes variant entre 1,4 et 1,7. blessures liées à une chute, ceux-ci pasChez les femmes, nous avons observé une sant de 61,0 (IC à $95 \%: 55,2$ à 66,9) pour augmentation constante des taux de 1000 personnes en 2005 à 72,4 (IC à
$95 \%: 63,9$ à 80,9) pour 1000 personnes en 2013 ( $p=0,03$ pour 2013 par rapport à 2005). Par contre, tandis qu'une 
augmentation marquée a été observée chez les hommes entre 2005 et 20092010, les taux passant de 34,9 (IC à $95 \%$ : 30,2 à 39,6) pour 1000 personnes à 46,3 (IC à $95 \%$ : 39,5 à 53,1) pour 1000 personnes ( $p=0,007$ ), il y a eu une légère diminution, non significative, entre 2009-2010 et 2013, le taux passant à 42,7 (IC à $95 \%: 35,0$ à 50,4) pour 1000 personnes en 2013. Contrairement à ce qui a été observé chez les femmes, la différence dans les taux observés chez les hommes entre l'échantillon de 2005 et celui de 2013 n'était pas significative sur le plan statistique $(p=0,10)$.

La figure 3 présente les taux de blessures liées à une chute par groupe d'âge et par année d'enquête. De manière générale, les taux de blessures liées à une chute ont augmenté avec l'âge pour toutes les années d'enquête, cette augmentation ayant été plus marquée chez les 80 à 84 ans et les 85 à 89 ans. Entre 2005 et 2013, une tendance importante à la hausse a été observée chez les 65 à 69 ans et chez les 70 à 74 ans ( $p=0,02$ pour les deux groupes), tandis qu'un déclin non significatif a été noté chez les plus âgés ( 90 ans et plus). En outre, si en 2005 le taux de blessures liées à une chute observé chez les aînés de 90 ans ou plus équivalait à 2,5 fois le taux observé chez ceux de 65 à 69 ans (102,8 contre 40,6 pour 1000 personnes), il a diminué ensuite, passant à 2,0 en 2009-2010 (100,6 contre 49,8 pour 1000 personnes), puis à 1,5 en $2013(83,8$ contre 55,4 pour 1000 personnes).

\section{Caractéristiques des blessures}

Les figures 4 et 5 , fondées sur les données combinées des échantillons de 2005, de 2009-2010 et de 2013, présentent la proportion de blessures liées à une chute, respectivement par type de blessure et par partie du corps affectée. Dans la majorité des cas, la chute a provoqué une cassure ou une fracture (37\%), une entorse ou une foulure $(27 \%)$ ou une éraflure, une meurtrissure ou une cloque $(17 \%)$. Les parties du corps les plus souvent affectées étaient l'épaule ou le bras (16\%), le genou ou la partie inférieure de la jambe (13\%), ou la cheville ou le pied (11\%). Bien que les termes utilisés dans les questionnaires
FIGURE 1

Nombres et taux de blessures liées à une chute, adultes de 65 ans et plus, Canada, ESCC 2005, 2009-2010 et 2013

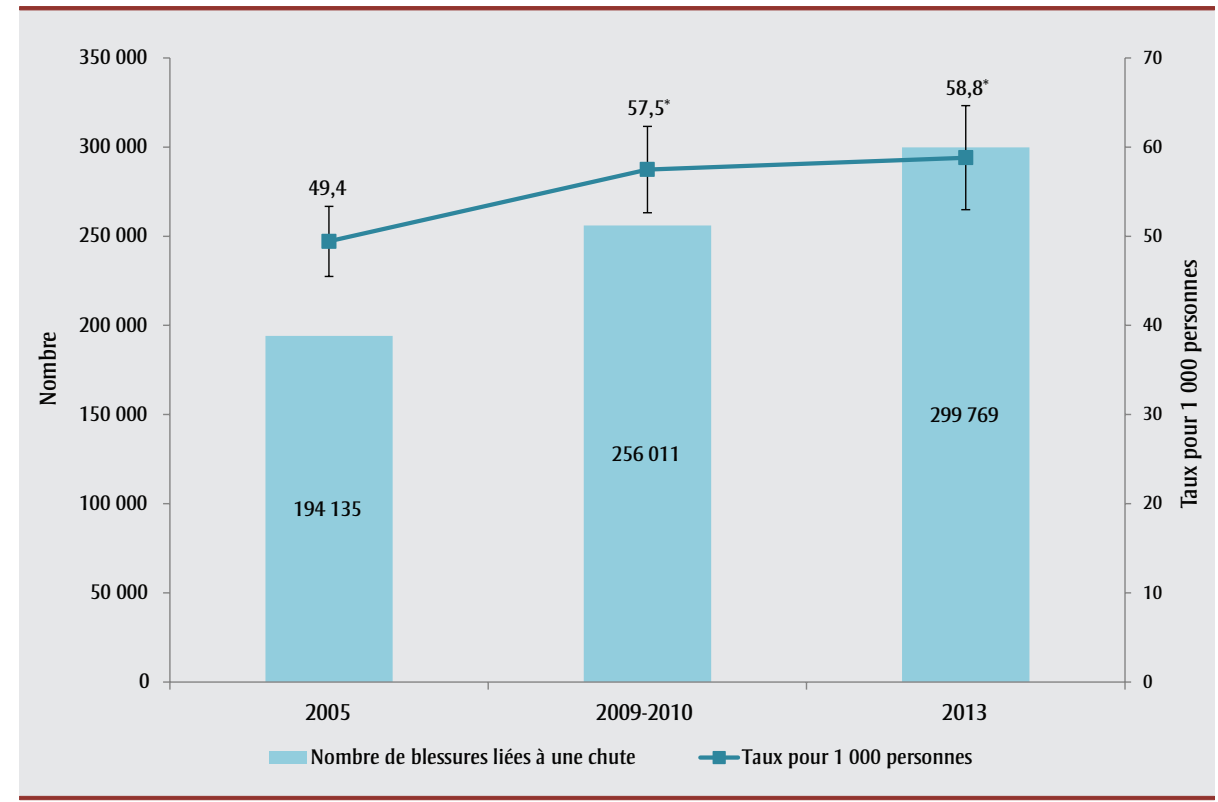

Source : Enquête sur la santé dans les collectivités canadiennes (2005, 2009-2010 et 2013), Statistique Canada. Abréviation : ESCC, Enquête sur la santé dans les collectivités canadiennes.

Remarques : Les nombres et les taux (pour 1000 personnes) de blessures liées à une chute ont été calculés à l'aide de poids d'échantillonnage. Les barres d'erreur correspondent à des intervalles de confiance à $95 \%$ des taux de blessures liées à une chute, calculés à l'aide de la méthode bootstrap.

* Valeur très différente de l'estimation de $2005(p<0,05)$ d'après la comparaison des proportions à l'aide du test $Z$.

\section{FIGURE 2}

Taux de blessures liées à une chute selon le sexe, adultes de 65 ans et plus, Canada, ESCC 2005, 2009-2010 et 2013

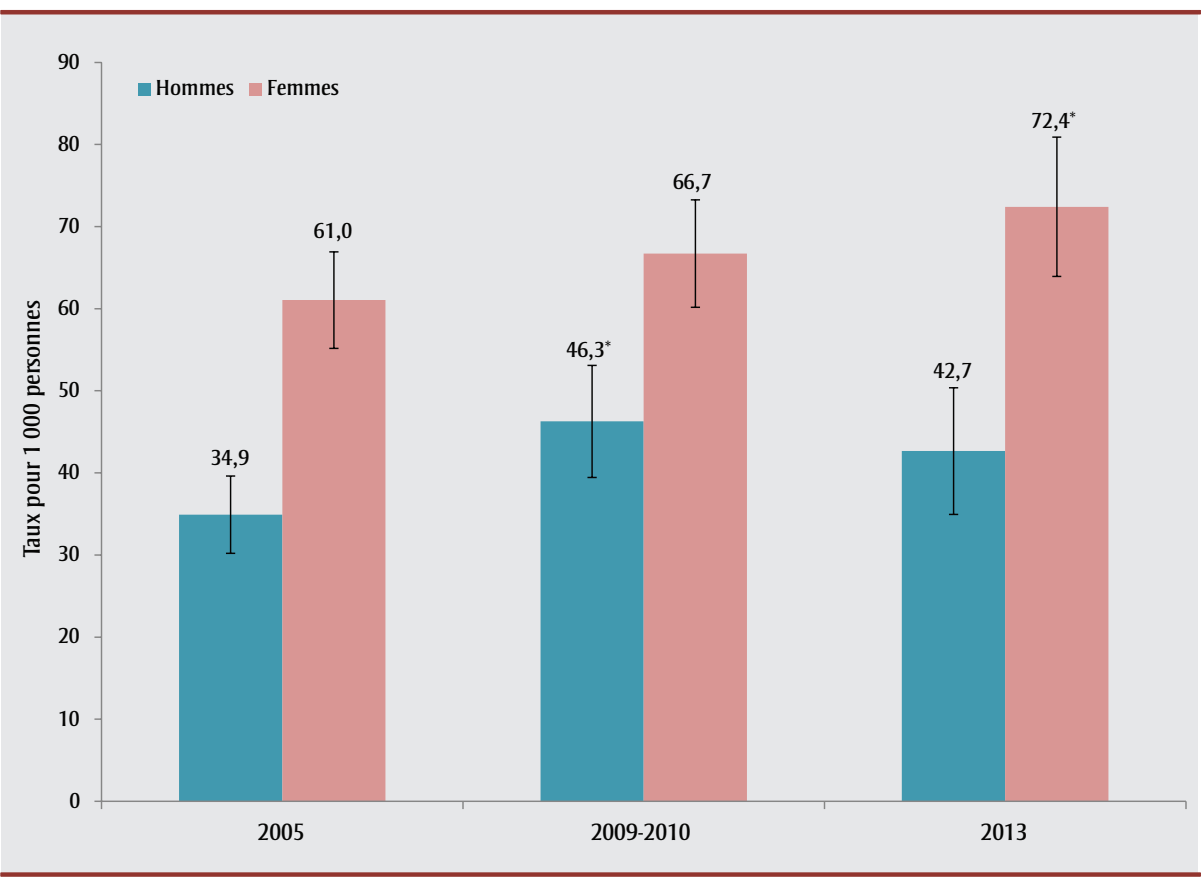

Source : Enquête sur la santé dans les collectivités canadiennes (2005, 2009-2010 et 2013), Statistique Canada. Abréviation : ESCC, Enquête sur la santé dans les collectivités canadiennes.

Remarques : Les taux de blessures liées à une chute ont été calculés pour 1000 personnes à l'aide de poids d'échantillonnage. Les barres d'erreur correspondent à des intervalles de confiance à $95 \%$ calculés à l'aide de la méthode bootstrap.

* Valeur très différente de l'estimation de $2005(p<0,05)$ d'après la comparaison des proportions à l'aide du test $Z$. 
FIGURE 3

Taux de blessures liées à une chute par groupe d'âge, adultes de 65 ans et plus, Canada, ESCC 2005, 2009-2010 et 2013

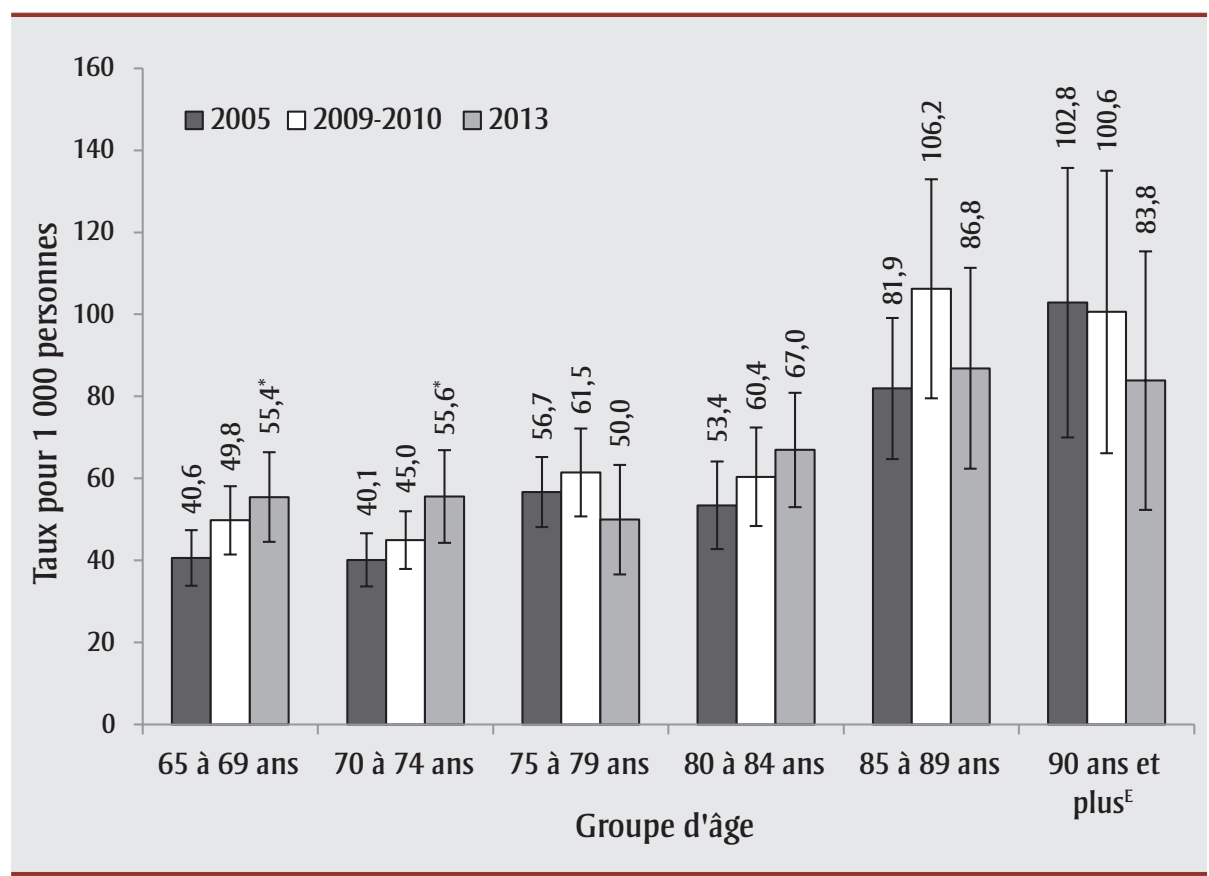

Source : Enquête sur la santé dans les collectivités canadiennes (2005, 2009-2010 et 2013), Statistique Canada. Abréviation : ESCC, Enquête sur la santé dans les collectivités canadiennes.

Remarques : Les taux de blessures liées à une chute ont été calculés pour 1000 personnes à l'aide de poids d'échantillonnage. Les barres d'erreur correspondent à des intervalles de confiance à $95 \%$ calculés à l'aide de la méthode bootstrap.

${ }^{E}$ Les estimations pour les échantillons de 2009-2010 et de 2013 étant liées à une variabilité d'échantillonnage élevée (c.-à-d. que le coefficient de variation se situe entre 16,6 \% et 33,3\%), ces données doivent être interprétées avec prudence. * Valeur très différente de l'estimation de $2005(p<0,05)$ d'après la comparaison des proportions à l'aide du test $\mathrm{Z}$.

de 2009-2010 et de 2013 aient légèrement changé par rapport aux termes utilisés dans le questionnaire de 2005 (par exemple, la catégorie « Entorse ou foulure » a été formulée avec plus de précision dans les questionnaires de 2009-2010 et de 2013 : « Entorse ou foulure [incluant les ligaments et les muscles déchirés »]), nous n’avons observé aucune différence significative entre les années d'enquête dans les proportions des catégories visées par ces changements (données non présentées).

La figure 6, fondée sur les données combinées des échantillons de 2009-2010 et de 2013, présente la proportion de blessures liées à une chute selon le type d'activité à l'origine de la chute, les données de l'échantillon de 2005 ayant été exclues en raison des différences dans le classement des types d'activités. Les blessures attribuables à une chute se sont produites dans la plus grande proportion des cas lorsque la personne a glissé, trébuché, fait un faux pas ou perdu l'équilibre, alors qu'elle marchait sur une surface non enneigée ou glacée (45\%), une surface enneigée ou glacée (16\%) ou qu'elle montait ou descendait un escalier ou des marches (12\%). Les autres répondants ont déclaré avoir fait une chute en raison de problèmes de santé (p. ex. perte de conscience, faiblesse, étourdissement, dislocation d'une hanche ou d'un genou, crise d'épilepsie) (7\%), en tombant d'un meuble ou en montant sur un meuble (p. ex. lit, chaise) (6 \%), durant la pratique d'un sport ou un exercice physique (5\%) ou depuis une position élevée (4\%).

Parmi les aînés de l'échantillon de 2005 ayant déclaré avoir subi une blessure liée à une chute, 133958 (69 \%) ont dit avoir consulté un professionnel de la santé dans les 48 heures suivant la blessure. Ils ont été 163571 à le faire en 2009-2010 et 198164 en 2013, mais dans des proportions légèrement plus faibles (64\% en 2009-2010 et $66 \%$ en 2013). D'après les données combinées des trois années d'enquête, la majorité des personnes qui ont cherché à obtenir des soins médicaux en raison d'une blessure liée à une chute se sont rendues au service d'urgence d'un hôpital (71 \%), au cabinet d'un médecin (15\%), à une clinique $(6 \%)$ ou à la clinique externe d'un hôpital (5\%). Moins de $5 \%$ ont déclaré avoir cherché à obtenir des soins médicaux dans un centre de santé communautaire ou là où la blessure est survenue. En outre, plus du quart des répondants ayant indiqué s'être rendus dans un établissement de soins médicaux pour une blessure liée à une chute ont indiqué avoir été hospitalisés pour la nuit. Des tendances similaires ont été observées pour toutes les années d'enquête (données non présentées).

\section{Analyse}

À l'aide des données des ESCC de 2005, de 2009-2010 et de 2013, nous avons dressé un profil national actualisé des blessures attribuables à une chute chez les Canadiens âgés de 65 ans et plus et nous en avons analysé les tendances.

Entre 2005 et 2013, le nombre d'aînés canadiens ayant subi une blessure en raison d'une chute a augmenté de $50 \%$, ce qui concorde avec les tendances au vieillissement de la population ${ }^{21}$. De plus, l'augmentation des taux de blessures liées à une chute coïncide avec l'augmentation déjà constatée entre 2003 et 2009-2010 au Canada ${ }^{7}$ ainsi qu'avec les tendances observées aux États-Unis ${ }^{29,30}$, aux Pays-Bas ${ }^{31}$ et en Austra$\operatorname{lie}^{32,33}$. Alors que la majorité de ces études se sont limitées aux blessures traitées dans un service d'urgence ou ayant nécessité une hospitalisation, notre étude présente les tendances concernant les blessures autodéclarées par suite d'une chute qu'un traitement médical ait été demandé ou non.

Nous avons constaté, à la suite d'autres études $^{29-36}$, que les taux de blessures liées à une chute étaient considérablement plus élevés chez les femmes plus âgées que chez les hommes plus âgés, et ce, pour toutes les années d'enquête. La perte de densité minérale osseuse suivant la ménopause 
FIGURE 4

Proportion de blessures liées à une chute par type de blessure, adultes de 65 ans et plus, Canada, ESCC 2005, 2009-2010 et 2013

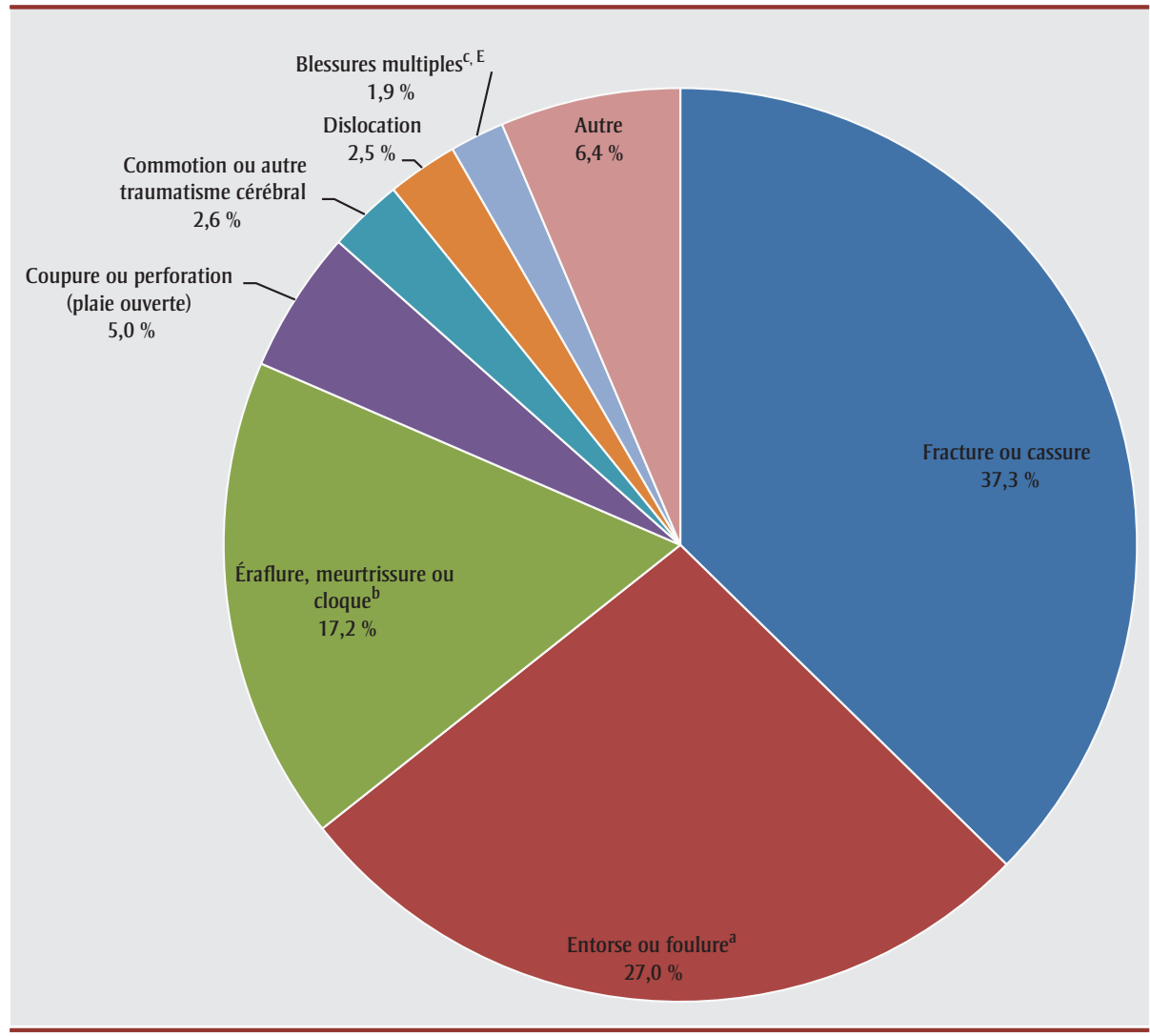

Source : Enquête sur la santé dans les collectivités canadiennes (enquêtes de 2005, 2009-2010 et 2013 combinées), Statistique Canada.

Abréviation : ESCC, Enquête sur la santé dans les collectivités canadiennes.

Remarque : Les termes utilisés dans les questionnaires de 2009-2010 et de 2013 pour certaines catégories ont légèrement changé par rapport au questionnaire de 2005 (voir ci-dessous), mais aucune différence significative n'a été observée dans les proportions de ces catégories entre les différentes enquêtes.

a Catégorie nommée « Entorse ou foulure » dans le questionnaire de 2005 et « Entorse ou foulure (incluant les ligaments et les muscles déchirés)» dans les questionnaires de 2009-2010 et de 2013.

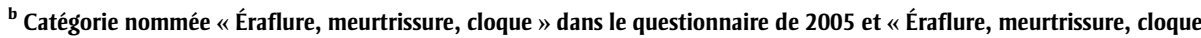
(incluant les blessures multiples mineures) » dans les questionnaires de 2009-2010 et de 2013.

c Catégorie nommée «Blessures multiples " dans le questionnaire de 2005 et «Blessures multiples (excluant les blessures multiples mineures) » dans les questionnaires de 2009-2010 et de 2013.

${ }^{E}$ L'estimation étant liée à une variabilité d'échantillonnage élevée (c.à-d. que le coefficient de variation se situe entre 16,6\% et $33,3 \%$ ), ces données doivent être interprétées avec prudence.

pourrait prédisposer les femmes à un risque accru de fracture ${ }^{37-39}$. Les disparités entre les sexes en ce qui concerne les taux de blessures attribuables à une chute pourraient également refléter les différences entre hommes et femmes quant à la force des membres inférieurs, à des problèmes de santé chroniques ainsi qu'à des facteurs liés au mode de vie ou au comportement ${ }^{40}$.

Une étude fondée sur les données recueillies dans le cadre de l'ESCC Vieillissement en santé a révélé que les facteurs de risque de chute diffèrent entre hommes et femmes, qu'ils soient sociodémographiques, liés au mode de vie et au comportement ou médicaux $^{41}$. Par exemple, le fait d'être veuf, séparé ou divorcé, d'avoir un niveau de scolarité plus élevé et de présenter un trouble de la vue ont été associés à un risque élevé de chute chez les hommes, tandis que chez les femmes, un âge avancé, un faible revenu, la consommation d'alcool ou celle de médicaments ainsi que le fait de souffrir de diabète ou d'ostéoporose ou de présenter un nombre plus élevé d'affections comorbides augmentaient le risque de chute $^{41}$. Il convient de noter que, bien que les femmes présentent un risque plus élevé de chute et de blessure liée à une chute, les taux de mortalité liés aux chutes sont plus élevés chez les hommes ${ }^{7,42,43}$, qui sont susceptibles de subir des blessures plus graves compte tenu des circonstances ou des événements entourant leur chute ${ }^{35}$.

Alors que le taux de blessures liées à une chute a augmenté de façon constante chez les femmes au fil du temps, nous avons observé un sommet chez les hommes en 2009-2010, suivi d'un déclin léger mais non significatif pour la dernière année d'enquête, lequel pourrait avoir contribué en partie à la stabilisation du taux global. Cette observation tranche avec les tendances observées entre 2003 et 2009-2010, les taux ayant alors augmenté de façon constante pour les deux sexes ${ }^{7}$.

Bien qu'il soit difficile d'établir des comparaisons directes compte tenu de différences dans les sources de données et les périodes examinées, les études déjà citées menées dans d'autres pays ont révélé des tendances à la hausse similaires chez les deux sexes ${ }^{29,30}$, voire une augmentation plus marquée chez les hommes que chez les femmes ${ }^{31-33}$. Les raisons du déclin (ou de la stabilisation) que nous avons observé récemment chez les hommes ne sont pas claires et pourraient être associées à des changements dans les comportements à risque et dans la perception du risque (p. ex. prendre des précautions supplémentaires au moment d'utiliser une échelle, marcher en étant plus attentif à l'environnement) ${ }^{6,7,44}$. Cependant, nous avons besoin d'années supplémentaires de données pour évaluer les tendances à long terme et déterminer si les taux de blessures liées à une chute diminuent réellement chez les hommes plus âgés au Canada.

Laugmentation du risque de chute avec l'âge a été largement prouvée $29-31,34-36$ et peut être attribuée au déclin des fonctions physiques, sensorielles et cognitives causé par l'âge, de même qu'à l'augmentation des comorbidités $^{43,45}$. En outre, nos résultats indiquent que, bien que les aînés plus âgés (85 ans et plus) continuent à présenter des taux plus élevés de blessures liées à une chute que les aînés plus jeunes, l'écart entre les groupes semble se resserrer avec le 
FIGURE 5

Proportion de blessures liées à une chute selon la partie du corps atteinte, adultes de 65 ans et plus, Canada, ESCC 2005, 2009-2010 et 2013

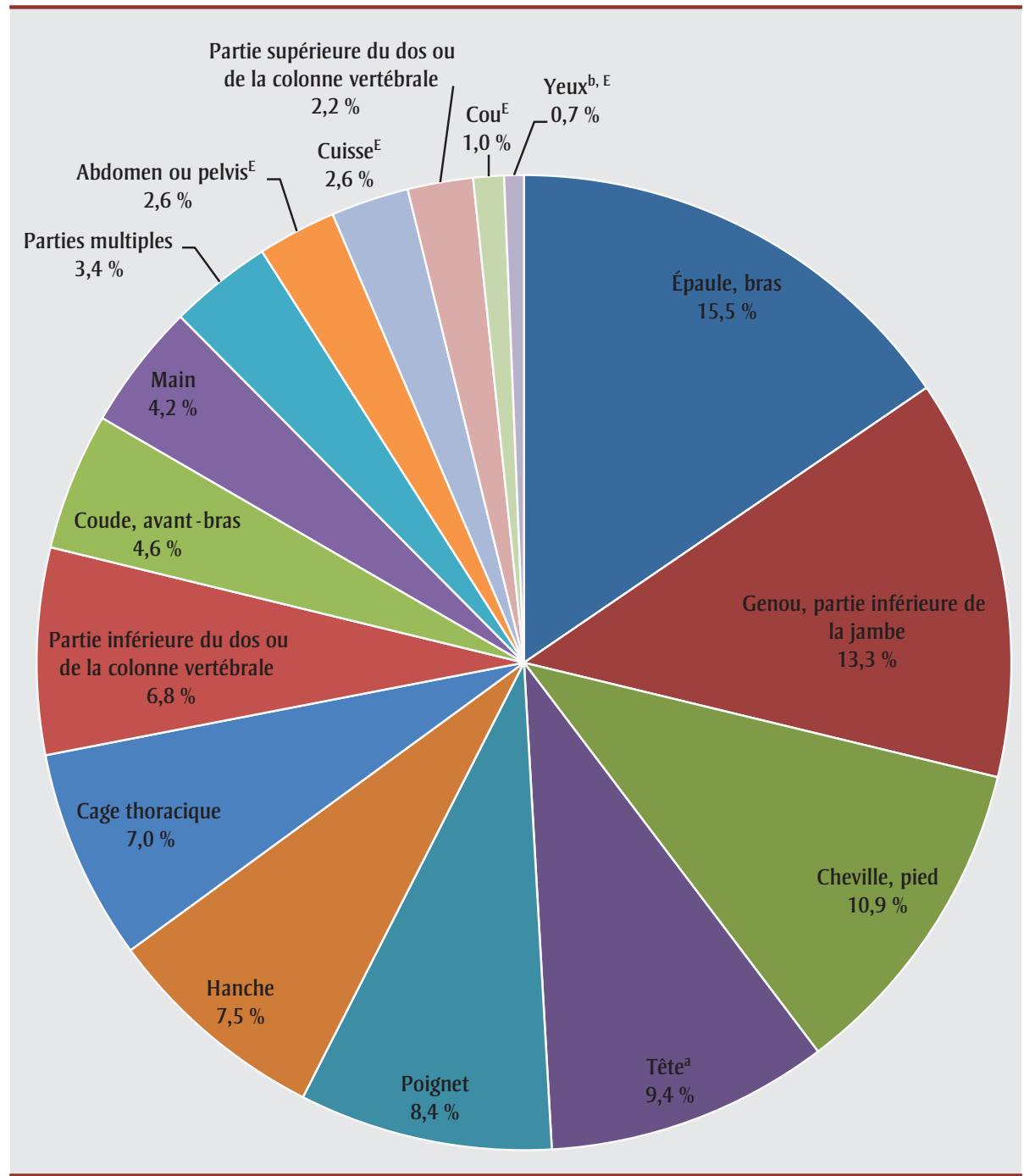

Source : Enquête sur la santé dans les collectivités canadiennes (enquêtes de 2005, 2009-2010 et 2013 combinées), Statistique Canada.

Abréviation : ESCC, Enquête sur la santé dans les collectivités canadiennes.

Remarque : Les termes utilisés dans les questionnaires de 2009-2010 et de 2013 pour certaines catégories ont légèrement changé par rapport au questionnaire de 2005 (voir ci-dessous), mais aucune différence significative n'a été observée dans les proportions de ces catégories entre les différents sondages.

a Catégorie nommée « Tête (excluant les yeux) » dans le questionnaire de 2005, et « Tête (incluant les os faciaux) » dans les questionnaires de 2009-2010 et de 2013.

b Catégorie nommée « Yeux » dans le questionnaire de 2005, et « Yeux (excluant une fracture d'un os facial autour d'un oil) » dans les questionnaires de 2009-2010 et de 2013.

${ }^{E}$ L'estimation est liée à une variabilité d'échantillonnage élevée (c.-à-d. que le coefficient de variation se situe entre $16,6 \%$ et $33,3 \%$ ) - ces données doivent être interprétées avec prudence.

temps. Le fait que les aînés plus jeunes mènent une vie plus active que par le passé et qu'ils consacrent plus de temps à des activités susceptibles d'accroître leur risque de chute pourrait expliquer cette tendance $^{29,46}$. Toutefois, ces tendances reposent fort probablement sur l'interaction complexe de plusieurs facteurs de risque de chute : modifications liées à l'âge dans le mode de vie et le comportement, problèmes de santé et utilisation de médicaments ${ }^{47}$. De plus, des déclins dans les taux de blessures liées à une chute ont aussi été enregistrés récemment chez les plus âgés (80 ans ou plus) en Finlande ${ }^{48}$ et pourraient témoigner d'améliorations dans leurs capacités fonctionnelles et dans leurs conditions de vie, quoique une évaluation plus approfondie des tendances soit nécessaire. À l'opposé, des études menées aux États-Unis ${ }^{30}$ et en Australie $^{33}$ ont fait état d'augmentations nettement plus marquées des taux chez les plus âgés par rapport aux plus jeunes.

Le fait que les fractures forment la plus grande proportion de blessures liées à une chute concorde avec les résultats des études portant sur les blessures liées à une chute ayant nécessité une hospitalisation $^{31,32}$ ou traitées dans un service d'urgence $^{35}$. Cela montre l'importance de la santé des os dans la prévention des fractures attribuables à une chute chez les aînés, en particulier compte tenu de la prévalence élevée de l'ostéoporose chez les femmes plus âgées ${ }^{7,49}$. Les circonstances entourant la survenue de blessures liées à une chute chez les aînés sont similaires à celles déclarées aux ÉtatsUnis $^{36}$, la majorité s'étant produites lorsque la personne a glissé, trébuché, fait un faux pas ou perdu l'équilibre. Cependant, la proportion relativement importante de blessures liées à une chute chez les aînés ayant marché sur une surface glacée ou enneigée est préoccupante et souligne le rôle que jouent les conditions météorologiques dans le risque de chute au Canada ${ }^{50}$. Nos résultats offrent aussi une illustration du fardeau qu'imposent les blessures liées à une chute sur le système de santé : plus des deux tiers des aînés canadiens ayant subi une blessure liée à une chute ont demandé des soins médicaux, le plus souvent au service d'urgence d'un hôpital. Avec le vieillissement de la population, on s'attend à une augmentation considérable du recours aux soins de santé dans les prochaines décennies en raison de blessures liées à une chute ainsi qu'à une augmentation des coûts qui leur sont associés.

\section{Forces et limites}

À notre connaissance, il s'agit de la première étude évaluée par les pairs sur les tendances relatives aux blessures autodéclarées à la suite d'une chute chez les aînés canadiens.

Le fait que nous ayons utilisé une enquête de population bien validée, qui a fourni un portrait national des blessures liées à une chute représentatif de la population des aînés canadiens vivant dans la collectivité, 


\section{FIGURE 6}

Proportion de blessures liées à une chute par type d'activité, adultes de 65 ans et plus, Canada, ESCC 2009-2010 et 2013

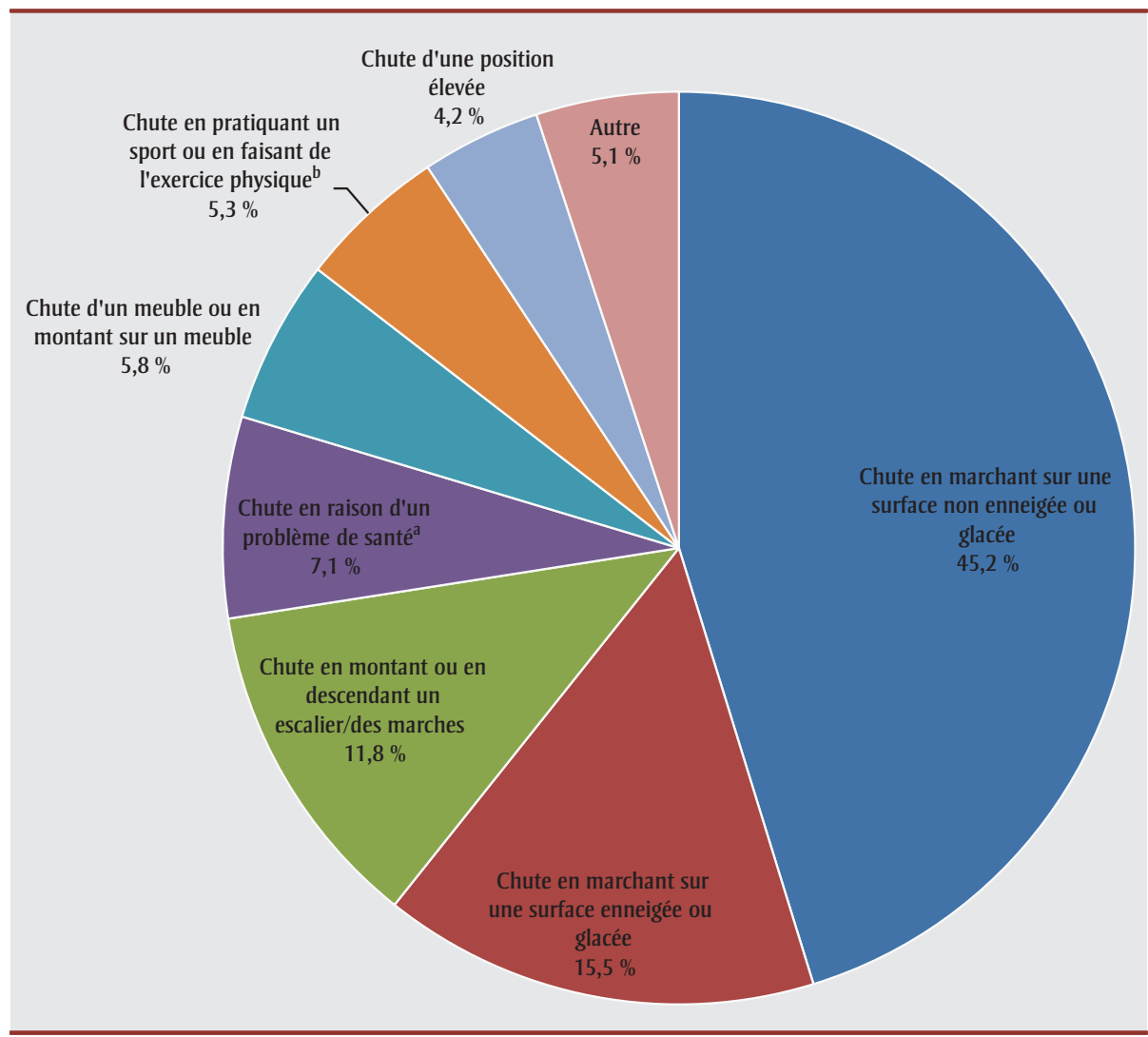

Source : Enquête sur la santé dans les collectivités canadiennes (enquêtes de 2009-2010 et de 2013 combinées), Statistique Canada.

Abréviation : ESCC, Enquête sur la santé dans les collectivités canadiennes.

Remarque : Les données de l'échantillon auprès duquel l'ESCC de 2005 a été menée n'ont pas été incluses dans l'analyse en raison de différences dans la classification des activités comparativement aux enquêtes de 2009-2010 et de 2013.

a Les exemples comprenaient perte de conscience, faiblesse, étourdissement, dislocation d'une hanche ou d'un genou, crise d'épilepsie.

${ }^{b}$ Cette catégorie comprenait le patin, le ski et la planche à neige.

constitue l'une des principales forces de cette étude. Loin de se limiter à des observations sur les blessures ayant nécessité une hospitalisation ou une visite au service d'urgence, notre étude présente des estimations et des tendances relevant d'un spectre large de blessures liées à une chute.

Notre étude présente également plusieurs limites, à prendre en considération au moment d'interpréter les résultats. Premièrement, étant donnée la façon dont les questions ont été conçues dans l'ESCC, seules les données sur les blessures les plus graves subies par les répondants dans l'année précédant l'enquête ont été retenues. Autrement dit, les renseignements sur les chutes à l'origine de blessures moins graves que celles provoquées par certaines causes (p. ex. collisions routières) s'étant produites au cours de la même période n’ont pas été saisis, ce qui a vraisemblablement entraîné une sous-estimation du nombre et des taux de blessures liées à une chute. De façon similaire, l'enquête n'a pas recueilli d'information sur les chutes multiples subies par une même personne.

Deuxièmement, comme l'ESCC ne visait pas à recueillir de données annuelles sur les blessures pour l'ensemble des provinces et des territoires du Canada, notre étude des tendances nationales ne porte que sur un nombre limité de points de données (2005, 2009-2010 et 2013). Les changements susceptibles de s'être produits durant les périodes non visées par une enquête peuvent donc ne pas avoir été saisis. Néanmoins, la tendance généralement à la hausse des blessures liées à une chute dont nous avons fait état concorde avec ce qui a été signalé antérieurement ${ }^{7}$.

Troisièmement, le fait que l'ESCC repose sur des renseignements autodéclarés pourrait avoir donné lieu à un biais de mesure et à une mauvaise classification de nos résultats. Par exemple, les questions au sujet des blessures pourraient avoir été interprétées différemment par certains répondants. La question de la sous-déclaration des chutes en raison d'un biais lié à la désirabilité sociale (p. ex. refus d'admettre avoir fait une chute) ou de la difficulté à se rappeler avoir fait une chute, surtout chez la population plus âgée ${ }^{51}$, pourrait aussi se poser.

Quatrièmement, les taux de réponse peu élevés, en particulier dans la dernière année d'enquête (66,8 \% en 2013), pourraient avoir donné lieu à une généralisabilité limitée des résultats de l'étude. Pour réduire le biais qui pourrait être associé au taux de non-réponse (comme le refus de participer ou l'incapacité de contacter la personne ou le ménage sélectionné), Statistique Canada a ajusté les coefficients de pondération en redistribuant les personnes ou les ménages n'ayant pas répondu et présentant des caractéristiques similaires (p. ex. données géographiques, période de collecte, moment des tentatives de contact et nombre de tentatives ${ }^{24}$.

Enfin, nos résultats ne peuvent être généralisés qu'à une population d'aînés relativement en santé et vivant dans la collectivité, et ils ne s'appliquent pas à ceux vivant dans des établissements de santé, qui sont susceptibles de présenter un profil de risque de chute distinct et dont les circonstances et les conséquences liées aux blessures pourraient différer de celles de la population générale ${ }^{52}$.

\section{Conclusion}

Les blessures liées à une chute chez les aînés canadiens ont, comme dans d'autres pays industrialisés, augmenté au cours de la dernière décennie. Les données récentes laissent également penser qu'il y a dans les tendances des différences liées au sexe et à l'âge, et que cette augmentation est plus marquée chez les femmes et chez les aînés plus jeunes. Comme la 
population canadienne vieillit rapidement, il demeure essentiel de surveiller les tendances et les profils des blessures liées à une chute, tout comme le fardeau que représentent ces blessures sur le système de santé.

\section{Références}

1. Tinetti ME, Speechley M, Ginter SF. Risk factors for falls among elderly persons living in the community $\mathrm{N}$ Engl $\mathrm{J}$ Med. 1988;319(26):1701-1707.

2. O'Loughlin JL, Robitaille Y, Boivin JF, Suissa S. Incidence of and risk factors for falls and injurious falls among the community-dwelling elderly Am J Epidemiol. 1993;137(3):342-354.

3. Tromp AM, Plujim SM, Smit JH, Deeg DJ, Bouter LM, Lips P. Fall-risk screening test: a prospective study on predictors for falls in community-dwelling elderly $\mathrm{J}$ Clin Epidemiol. 2001;54(8):837-844.

4. Gill T, Taylor AW, Pengelly A. A populationbased survey of factors relating to the prevalence of falls in older people. Gerontology. 2005;51(5):340-345

5. Morrison A, Fan T, Sen SS, Weisenfluh L. Epidemiology of falls and osteoporotic fractures: a systematic review Clinicoecon Outcomes Res. 2013;5:9-18.

6. Pearson C, St-Arnaud J, Geran L. Comprendre le risque de chute chez les aînés et leur perception du risque. Coup d'œil sur la santé, octobre 2014. Ottawa (Ont.) : Statistique Canada; 2014 (Statistique Canada, ${ }^{\circ}$ 82-624-X).

7. Agence de la santé publique du Canada. Chutes chez les aînés au Canada : Deuxième rapport. Ottawa (Ont.) : Agence de la santé publique du Canada, 2014.

8. Gill TM, Murphy TE, Gahbauer EA, Allore HG. Association of injurious falls with disability outcomes and nursing home admissions in community-living older persons Am J Epidemiol. 2013;178(3):418-425.

9. Todd C, Skelton D. What are the main risk factors for falls among older people and what are the most effective interventions to prevent these falls? Health Evidence Network Report. Copenhagen (DM) : World Health Organization Regional Office for Europe; 2004
10. Wolinsky FD, Bentler SE, Liu L et collab. Recent hospitalization and the risk of hip fracture among older Americans J Gerontol A Biol Sci Med Sci. 2009;64 (2):249-55

11. Ioannidis G, Papaioannou A, Hopman WM et collab. Relation between fractures and mortality: results from the Canadian Multicentre Osteoporosis Study CMAJ. 2009;181 (5):265-271.

12. Stel VS, Smit JH, Pluijm SM, Lips P. Consequences of falling in older men and women and risk factors for health service use and functional decline Age Ageing. 2004;33(1):58-65.

13. Scott V, Pearce M, Pengelly C. Rapport technique : Les blessures résultant de chutes des Canadiens âgés de 65 ans et plus portant sur l'analyse des données de l'Enquête sur la santé dans les collectivités canadiennes, Cycle 2.1 présentée dans le Rapport sur les chutes des aînés au Canada. Ottawa (Ont.) : Agence de la santé publique du Canada; 2005.

14. Tinetti ME, Kumar C. The patient who falls: "it's always a trade-off" JAMA.2010;303 (3):258-266.

15. Scuffham P, Chaplin S, Legood R. Incidence and costs of unintentional falls in older people in the United Kingdom J Epidemiol Community Health. 2003;57(9):740-744.

16. Stevens JA, Corso PS, Finkelstein EA, Miller TR. The costs of fatal and non-fatal falls among older adults Inj Prev. 2006;12 (5):290-295.

17. Watson WL, Clapperton AJ, Mitchell RJ. The cost of fall-related injuries among older people in NSW, 2006-07 N S W Public Health Bull. 2011;22(3-4):55-59.

18. Hartholt KA, Polinder S, van der Cammen $\mathrm{TJ}$ et collab. Costs of falls in an ageing population: a nationwide study from the Netherlands (2007-2009) Injury. 2012;43 (7):1199-1203

19. Sauve-qui-pense. The economic burden of injury in Canada. Toronto (Ont.) : Sauvequi-pense; 2009.

20. Statistique Canada. La population canadienne en 2011 : âge et sexe. Ottawa (Ont.) : Statistique Canada; $2012 \quad\left(\mathrm{n}^{\circ}\right.$ 98-311X2011001 au catalogue).
21. Statistique Canada. Projections démographiques pour le Canada, les provinces et les territoires, 2009 à 2036. Ottawa (Ont.) : Statistique Canada; 2010 (n ${ }^{\circ}$ 91-520-X au catalogue).

22. Statistique Canada. Enquête sur la santé dans les collectivités canadiennes (ESCC) Cycle 3.1 (2005) : Fichier de microdonnées à grande diffusion (FMGD) : Guide de l'utilisateur. Ottawa (Ont.) : Statistique Canada; 2006.

23. Statistique Canada. Enquête sur la santé dans les collectivités canadiennes (ESCC) - Composante annuelle : Guide de l'utilisateur : Fichier de microdonnées de 2010 et 2009-2010. Ottawa (Ont.) : Statistique Canada; 2011.

24. Statistique Canada. Enquête sur la santé dans les collectivités canadiennes (ESCC) Composante annuelle : Guide de l'utilisateur : Fichier de microdonnées de 2013. Ottawa (Ont.) : Statistique Canada; 2014.

25. Yeung CW, Thomas S. Imputation for the Canadian Community Health Survey [L'imputation du revenu pour l'Enquête sur la santé dans les collectivités canadiennes]. Working Paper (Methodology Branch). Ottawa (Ont.) : Statistique Canada; 2013 (n 11-619E n 2013-003 au catalogue].

26. Rao JN, Scott AJ. On chi-squared tests for multiway contingency tables with cell proportions estimated from survey data, Ann Statist. 1984;12(1):46-60.

27. Thomas S, Wannell B. Combiner les cycles de l'Enquête sur la santé dans les collectivités canadiennes Rapports sur la santé. 2009;20(1):59-65.

28. Statistique Canada. BootVar : guide de l'usager (BootVar 3.1 - Version SAS). Ottawa (Ont.) : Statistique Canada; 2010.

29. Hartholt KA, Stevens JA, Polinder S, van der Cammen TJ, Patka P. Increase in fall-related hospitalizations in the United States, 20012008. J Trauma. 2011;71(1):255-258.

30. Orces $\mathrm{CH}$, Alamgir $\mathrm{H}$. Trends in fall-related injuries among older adults treated in emergency departments in the USA Inj Prev. 2014;20(6):421-423.

31. Hartholt KA, van der Velde N, Looman CW et collab. Trends in fall-related hospital admissions in older persons in the Netherlands Arch Intern Med. 2010;170(10):905-911. 
32. Watson WL, Mitchell R. Conflicting trends in fall-related injury hospitalisations among older people: variations by injury type. Osteoporos Int. 2011;22(10):2623-2631.

33. Bradley C. Trends in hospitalisations due to falls by older people, Australia 1999-00 to 2010-11. Injury research and statistics series no. 84. Canberra (AU) : Australian Institute of Health and Welfare; 2013 (INJCAT $n^{\circ}$ 160).

34. Peel NM, Kassulke DJ, McClure RJ. Population based study of hospitalised fall related injuries in older people. Inj Prev. 2002;8 (4):280-283

35. Stevens JA, Sogolow ED. Gender differences for non-fatal unintentional fall related injuries among older adults. Inj Prev. 2005; 11(2):115-119.

36. Schiller JS, Kramarow EA, Dey AN. Fall injury episodes among noninstitutionalized older adults: United States, 2001-2003. Advance data from vital and health statistics; no. 392. Hyattsville (MD) : National Center for Health Statistics; 2007 (PHS n 2007-1250).

37. Siris ES, Miller PD, Barrett-Connor E et collab. Identification and fracture outcomes of undiagnosed low bone mineral density in postmenopausal women: results from the National Osteoporosis Risk Assessment JAMA. 2001;286(22):2815-2822.

38. Stone KL, Seeley DG, Lui LY et collab. BMD at multiple sites and risk of fracture of multiple types: long-term results from the Study of Osteoporotic Fractures J Bone Miner Res. 2003;18(11):1947-1954.

39. Cranney A, Jamal SA, Tsang JF, Josse RG, Leslie WD. Low bone mineral density and fracture burden in postmenopausal women CMAJ. 2007;177(6):575-580.

40. Duckham RL, Procter-Gray E, Hannan MT, Leveille SG, Lipsitz LA, Li W. Sex differences in circumstances and consequences of outdoor and indoor falls in older adults in the MOBILIZE Boston cohort study BMC Geriatr. 2013;13:133.

41. Chang VC, Do MT. Risk factors for falls among seniors: implications of gender Am J Epidemiol. 2015;181(7):521-531.

42. Stevens JA, Ryan G, Kresnow M. Fatalities and injuries from falls among older adults United States, 1993-2003 and 2001-2005 MMWR. 2006;55(45):1221-1224.
43. Organisation mondiale de la santé (OMS). WHO global report on falls prevention in older age. Genève $(\mathrm{CH})$ : OMS; 2007. Téléchargeable en anglais à partir de la page : http://www.who.int/ageing/projects/falls_ prevention_older_age/en/\#.

44. Yuen HK, Carter RE. A measure of fall risk behaviors and perceptions among communitydwelling older adults J Allied Health. 2006; 35(4):e276-e297.

45. Ambrose AF, Paul G, Hausdorff JM. Risk factors for falls among older adults: a review of the literature Maturitas. 2013;75(1):51-61.

46. Victorino CC, Gauthier AH. Are Canadian seniors becoming more active? Empirical evidence based on time-use data.Can $\mathrm{J}$ Aging. 2005;24(1):45-56.

47. Deandrea S, Lucenteforte E, Bravi F, Foschi R, La Vecchia C, Negri E. Risk factors for falls in community-dwelling older people: a systematic review and meta-analysis Epidemiology. 2010;21(5):658-668.

48. Korhonen N, Niemi S, Palvanen M, Parkkari J, Sievänen H, Kannus P. Declining ageadjusted incidence of fall-induced injuries among elderly Finns Age Ageing. 2012;41 (1):75-79.

49. Cummings-Vaughn LA, Gammack JK. Falls, osteoporosis, and hip fractures Med Clin North Am. 2011;95(3):495-506.

50. Gao C, Abeysekera J. A systems perspective of slip and fall accidents on icy and snowy surfaces. Ergonomics. 2004;47(5):573-98.

51. Cummings SR, Nevitt MC, Kidd S. Forgetting falls The limited accuracy of recall of falls in the elderly. J Am Geriatr Soc.1988;36 (7):613-6.

52. Deandrea S, Bravi F, Turati F, Lucenteforte E, La Vecchia C, Negri E. Risk factors for falls in older people in nursing homes and hospitals. A systematic review and metaanalysis. Arch Gerontol Geriatr.2013;56(3): 407-15. 